\title{
Polarização da cárie dentária em adolescentes, na região sudoeste do Estado de São Paulo, Brasil
}

\author{
Polarization of dental caries in teen-agers \\ in the Southwest of the state of São Paulo, Brazil
}

Sílvia H elena de Carvalho Sales Peres ${ }^{1}$

Fábio Silva de Carvalho ${ }^{2}$

Cristiane Paz de Carvalho ${ }^{2}$

José Roberto de $M$ agal hães Bastos ${ }^{1}$

JoséRoberto Pereira Lauris ${ }^{1}$

${ }^{1}$ Departamento de

Odontopediatria, Ortodontia

eSaúde Coletiva,

Universidade deSão Paulo.

Alameda O ctávio Pinheiro

Brisola 9-75, Vila

Universitária. 17012-101

Bauru SP. shcperes@usp.br

${ }^{2}$ Faculdade deO dontologia

deBauru, Universidade de

São Paulo.

${ }^{3}$ Departamento de

Odontopediatria, Ortodontia

eSaúdeColetiva, Faculdade

deOdontologia deBauru,

Universidade de São Paulo.
Abstract In most Brazilian cities, oral health is still a challenge for the Brazilian Health System (SUS). The aim of this study was to analyze the prevalence of dental caries (DM FT index), thedifferences with regard to gender and geographic localization, Significant Caries Index and the percentages of caries-free adolescents in Itaí-SP Brazil, in 2006. Themethodology used wasin agree ment with the criteria established by the WHO. The sample was composed of 178 teen-agers, corresponding to $46 \%$ of the 12 -years-old registered in the schools of the city. O ne single examiner performed the examination. The Kappa test method was used with an agreement rater of 0.95 . The DM TF index was of $2.45, \mathrm{SiC}$ of 5.08 and $30 \%$ of the adolescents were caries-free. It was observed that $70 \%$ of caries cases were concentrated in $34 \%$ of the adolescents. The data obtained showed a polarization of dental caries in 12-years-old adolescents, but this polarization was not uniform. The problems are concentrated in a small portion of the population.

Key words Dental caries, DMF index, Dental health surveys
Resumo A saúdebucal na maioria dosmunicípios brasilei ros constitui ainda um grande desafio aos princípios doutrinários do SUS. Este estudo objetivou analisar a prevalência de cárie dentária (CPOD) eas diferenças quanto ao gênero elocalização geográfica, Significant Caries Index (SiC) e a porcentagem de livres de cárie no município de Itaí, São Paulo, em 2006. U tilizou-sea metodologia da O M S (1997), em uma população constituída por 390; destes, 178 adol escentes aos 12 anos de idade eque correspondem a $46 \%$ dos adolescentes matriculados nas escolas do município. 0 exame foi realizado por um único examinador. 0 teste kappa foi calculado com valor de concordância de 0,95. 0 índice CPOD foi de 2, 45, o SiC de 5, 08, e $30 \%$ dos indivíduos se apresentaram livres de cárie. Observou-se que 34\% dos adolescentes concentraram $70 \%$ da doença demonstrando a ocorrência da polarização da cárie dentária. Foram encontradas diferenças estatisticamentesignificantes na comparação do CPOD entre a localização geográfica e o mesmo não aconteceu com o gênero. Concluiu-se que está ocorrendo a polarização da cárie dentária em adolescentes, aos 12 anos, mas esta ocorrência não se apresenta de forma homogênea. 0 s problemas se intensificam em uma pequena parcela da população.

Palavras-chave Cárie dentária, Índice CPO, Le vantamentos de saúde bucal 
Introdução

A saúde bucal, na maioria dos municípios brasileiros, constitui ainda um grande desafio aos princípios doutrinários do SUS, principalmente no que se refere à universalização e à eqüidade do atendimento ${ }^{1}$.

A pesar dos esforços realizados, a cárie dentária ainda é considerada uma endemia mundial, principalmente entre crianças e adolescentes ${ }^{2}$ mesmo com o declínio que vem ocorrendo ao longo dos anos; portanto, necessita de medidas individuais e coletivas, bem como estratégias preventivas para controlá-la e tornar suas seqüelas cada vez menos severas ${ }^{3}$.

No Brasil, com a introdução dos dentifrícios fluoretados no final da década de 1980, houve uma mudança no perfil da cárie dentária, ocorrendo a diminuição no índice CPOD e aumento no percentual deindivíduos livres de cárie4. Para $N$ arvai et al. ${ }^{5}$, a fluoretação das águas de abastecimento público, a adição de compostos fluoretados aos dentifrícios e a descentralização do sistema de saúde brasileiro são fatores que devem ser considerados para compreender o processo de declínio na experiência de cárie dentária em dentes permanentes de escolares brasileiros a partir do final do século XX.

Em 1986, foi realizado um levantamento epidemiológico a nível nacional, no qual o índice CPOD foi de 6,67 aos 12 anos de idade ${ }^{6}$, uma prevalência de cárie muito alta segundo a Organização Mundial da Saúde $(\mathrm{OMS})^{7}$, como pode ser observado na Tabela 1. Por outro lado, em 1996 observou-se uma queda de $54 \%$ no índice CPOD ao longo desses dez anos, atingindo 3,068. valor próximo à meta estabelecida para 0 ano 2000 pela OMS.

O conhecimento da situação epidemiológica na população éessencial para o planejamento ea execução de ações em saúde bucal, sendo o caminho correto de superação do atendimento indiscriminado da livre demanda, ou seja, do atendi- mento às pessoas que por sua própria iniciativa recorrem às unidades detratamento disponíveis ${ }^{9}$. Por isso a odontologia deve observar a distribuição dos agravos e das necessidades de tratamento dentário, em cada segmento de nossa sociedade, para promover a saúde bucal de um modo efetivo ${ }^{10}$. Segundo Roncalli ${ }^{11}$, o diagnóstico de saúde, proporcionado pela epidemiologia, está inserido dentro das estratégias globais de planejamento e de avaliação nos serviços de saúde. Ressaltando que não devem ocorrer momentos estanques ou anteriores às ações de saúde, mas 0 chamado "enfoque epidemiológico" dos serviços, que deve ser uma prática transversal e cotidiana. Além disso, os levantamentos epidemiológicos fornecem dados que possibilitam comparações no tempo e no espaço ${ }^{12}$.

A maior concentração dos serviços públicos de saúde na zona urbana compromete edificulta o acesso da população residente na zona rural aosatendimentos odontológicos eacredita-seque estes indivíduos possam representar um importante pólo de concentração dos agravos à saúde bucal ${ }^{10}$; desta forma, torna-se importante a investigação desta população para o conhecimento de sua realidade.

Lopes \& Bastos $^{13}$ realizaram uma análise da literatura científica, sobre a prevalência de cárie dentária nos gêneros masculino efeminino. Identificaram que existia uma maior prevalência de cárie dentária no gênero feminino devido à erupção precoce dos dentes neste grupo. Em estudo mais recente, Sales-Peres \& Bastos ${ }^{4}$ relataram algumas mudanças neste perfil em favor do gênero feminino, devido à maior conscientização ao autocuidado, realização melhor e por mais vezes ao dia do controle mecânico da placa. Por outro lado, destacaram que os meninos talvez tenham menor rigor com a própria saúde. Essa tendência deve ser melhor investigada para que futuras afirmações possam ser elaboradas.

O declínio da cárie dentária observado em vários países foi acompanhado pelo fenômeno

Tabela 1. Classificação do ataque de cárie dentária com base nos valores do CPO aos 12 anos de idade.

\begin{tabular}{cccccc}
\hline Prevalência & Muito baixa & Baixa & Média & Alta & Muito alta \\
\hline Índice CPO & 0,0 a 1,1 & 1,2 a 2,6 & 2,7 a 4,4 & 4,5 a 6,5 & $\geq 6,6$ \\
\hline
\end{tabular}

Fonte: OM S, 1994. 
da polarização, que consiste na concentração da maior parte das doenças e das necessidades de tratamento em uma pequena parcela da população ( 20 a 40\%), sendo que a maior parte das crianças apresenta-se livre de cárie $\left(40\right.$ a 60\% ) ${ }^{4,14-16}$. A polarização é um fenômeno que pode estar refletindo medidas de prevenção e controle da cárie, embasadas na estratégia populacional ${ }^{17}$. Passouse de uma situação de alta prevalência da doença para um cenário em que se constata uma grande porcentagem de indivíduos livres de cárie. Os valores cada vez mais baixos de CPOD aos 12 anos de idade encontrados indicam redistribuição de uma menor carga de doença. Além disso, cada vez mais a distribuição da cárie vai se afastando de uma distribuição uniforme, sendo notados níveis crescentes de desigualdade ${ }^{18}$. O bserva-seque a situação continua grave nas classes menos favorecidas eéa capacidadedeidentificar esses grupos, com alto índice de cárie dentária, queirá possibilitar o correto manuseio e a adoção de medidas e estratégias preventivas adequadas ${ }^{19}$.

O objetivo deste estudo foi identificar a prevalência da cárie dentária e o grupo $\mathrm{SiC}$, em adolescentes aos 12 anos de idade, analisando as variáveis gênero elocalização geográfica, no município de I taí, São Paulo.

\section{M aterial e método}

O município de Itaí localiza-sea 300 km da capital paulista, na região sudoeste do estado, pertencente ao DRS - VI (Departamento Regional de Saúde de Bauru). Segundo o Instituto Brasileiro de Geografia e Estatística (IBGE), apresenta uma população estimada em 2006 de 23.421 habitantes ${ }^{20}$. 0 município apresenta sistema de fluoretação das águas e, de acordo com dados da vigilância sanitária municipal, é feito o heterocontrole do flúor na água, apresentando nível satisfatório.

Este estudo foi aprovado pelo ComitêdeÉtica em Pesquisa em Seres Humanos da Faculdade de O dontologia de Bauru da Universidade de São Paulo (FOB-USP) no 56/2006, segundo a resolução n 196/96 do Conselho N acional de Saúde, e foi realizado após autorização dos diretores das escolas e dos pais ou responsáveis mediante preenchimento do termo de consentimento livre e esclarecido (TCLE).

A idade de 12 anos foi escolhida por se tratar de uma idade índice, permitindo comparações internacionais econtrole das ten dências deocorrência da cárie dentária ao longo do tempo.

O município de Itaí apresenta quatro escolas com alunos aos 12 anos de idade e todas estão localizadas na zona urbana. 0 número de adolescentes nesta idade foi obtido junto à secretaria das escolas, correspondendo a um total de 390. Registrou-se o local de residência do indivíduo durante o exame com o objetivo de investigar as diferenças das condições de saúde bucal entre os adolescentes que moravam nas zonas urbana e rural. Foram distribuídos os termos de consentimento livre e esclarecido a todos adolescentes regularmentematriculados nas escolas estaduais. 0 critério adotado para inclusão neste estudo foi a apresentação do TCLE assinado pelo pai ou responsável legal ea presença na escola no dia do levantamento epidemiológico. Foram examinados 178 indivíduos, correspondendo a $46 \%$ do universo de adolescentes aos 12 anos de idade, do município de Itaí, em 2006.

A presente pesquisa éum estudo do tipo transversal, caracterizado por fazer parte de um corte no fluxo histórico da doença, evidenciando as características apresentadas por ela no momento ${ }^{21}$.

Os adolescentes foram examinados durante os meses de junho ejulho de 2006 por um único examinador, cirurgião-dentista, que foi previamentecalibrado por um examinador "Gold Standard", visando garantir a uniformidade de interpretação, compreensão e aplicação dos critérios adotados ${ }^{12}$. Os dados coletados foram registrados por um anotador em fichas apropriadas para 0 estudo. Foram realizados reexames de aproximadamente $10 \%$ das crianças ${ }^{10}$ para medir a concordância intra-examinador, análise de kappa.

Os exames foram realizados no pátio das escolas, sob luz natural, com o examinador e 0 adolescente sentados em cadeiras e o material utilizado foi espelho bucal plano no 5 e a sonda CPI ("ball point").

Os índices utilizados para coleta dos dados obedeceram aos critérios recomendados pela OM S22, adaptados pela Faculdade de Saúde Pública da Universidade de São Paulo epelo Projeto SB Brasil ${ }^{23}$. A pós a análise do CPOD, procedeu-se ao cálculo do Significant Caries Index (SiC- média do CPOD para um terço do grupo com os maiores níveis da doença), para verificação dos casos de distribuição assimétrica. Foi analisado o percentual de adolescentes livres de cárie. 0 processamento dos dados foi realizado em planilha Excel eprocederam-sea sua descrição, análiseeapresentação das informações obtidas no estudo.

A análise estatística foi realizada por meio de freqüências relativas e absolutas, em gráficos e tabelas; utilizou-seo teste estatístico $\mathrm{M}$ ann-Whitney e adotou-se nível de significância de 5\%. 


\section{Resultados}

A análise do perfil epidemiológico de cárie dentária foi realizada avaliando-se o índice CPOD aos 12 anos de idade, o SiC e o percentual de adolescentes livres de cárie.

Foram reexaminados vinteadolescentes $(10 \%$ da amostra) para aferir a concordância intraexaminador eutilizou-seo teste estatístico kappa para análise eo valor obtido foi de 0,95 , considerado um indicativo de ótima concordância.

0 percentual de adolescentes livres de cárie neste estudo foi de $30 \%$ e o CPOD encontrado foi de 2,45. Avaliou-se o SiC desta população e o valor obtido foi de 5,08. Esses resultados estão demonstrados na Tabela 2.

Para comparações entre as variáveis testadas, utilizou-se o teste M ann-Whitney. A composição da amostra foi equilibrada quanto ao gênero, 55\% feminino com CPOD médio de 2,51 e $45 \%$ masculino com CPOD médio de 2,38; não houve diferença estatisticamente significante. Com relação à moradia, $75 \%$ dos adolescentes residem na zona urbana com CPOD médio de 2,21 e $25 \%$ residem na zona rural com CPOD médio de 3,18; observou-se diferença estatisticamente significante $(p=0,003)$. Quanto à representatividade dos componentes do CPOD, pode

Tabela 2. Índice CPOD, SiC índex e livres de cárie, em adolescentes aos 12 anos de idade. I taí, São Paulo, 2006.

\begin{tabular}{ccc}
\hline CPOD & SIC & Livres de cárie \\
\hline 2,45 & 5,08 & $30 \%$ \\
\hline
\end{tabular}

se observar que o restaurado " $\mathrm{O}$ " foi o que mais contribuiu para o valor encontrado do CPOD com $64 \%$, seguido do cariado "C" com $32 \%$ e o extraído "P" com 4\%. Na Tabela 3, estes dados podem ser visualizados, assim como a discriminação dos componentes do CPOD, com suas respectivas porcentagens.

O Gráfico 1 ilustra a distribuição da população estudada, relacionando-a ao valor do CPOD, com as respectivas porcentagens. Nota-se uma participação maior nos valores de CPOD 3 (16 \%) e CPOD 4 (18\%), e observa-se um declínio da curva até atingir CPOD 10 (1\%).

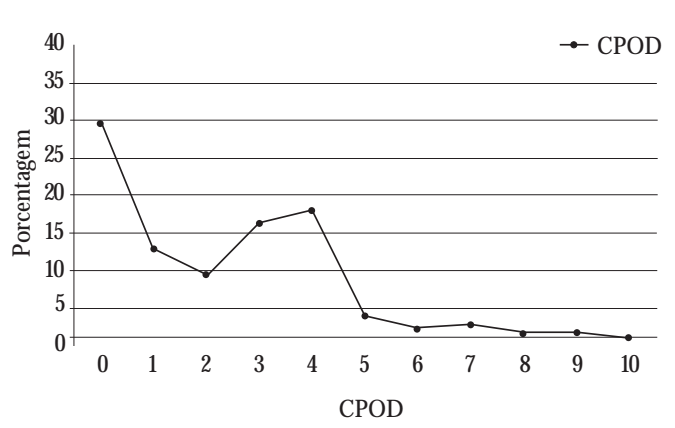

Gráfico 1. Curva de distribuição do CPOD, em valores percentuais em hebiatras aos 12 anos de idade. Itaí - SP, 2006.

Tabela 3. Índice CPOD e componentes em adolescentes aos 12 anos de idade, segundo o gênero e a moradia. Itaí, São Paulo, 2006.

\begin{tabular}{|c|c|c|c|c|c|}
\hline \multirow{2}{*}{$\begin{array}{l}\text { Condição } \\
\text { dentária }\end{array}$} & \multirow{2}{*}{$\begin{array}{c}\text { Amostra total } \\
\quad(100 \%) \\
n=178\end{array}$} & \multicolumn{2}{|c|}{ Gênero } & \multicolumn{2}{|c|}{ Moradia } \\
\hline & & $\begin{array}{c}\text { Masculino (45\%) } \\
n=80\end{array}$ & $\begin{array}{l}\text { Feminino (55\%) } \\
\quad \mathrm{n}=98\end{array}$ & $\begin{array}{c}\text { Urbana (75\%) } \\
n=133\end{array}$ & $\begin{array}{l}\text { Rural }(25 \%) \\
n=45\end{array}$ \\
\hline Cariados (C) & $32 \%$ & $42 \%$ & $25 \%$ & $34 \%$ & $29 \%$ \\
\hline Extraídos (P) & $4 \%$ & $4 \%$ & $5 \%$ & $4 \%$ & $5 \%$ \\
\hline Restaurados (0) & $64 \%$ & $54 \%$ & $70 \%$ & $62 \%$ & $66 \%$ \\
\hline CPOD & $2,45 \%$ & $2,38 \%$ & $2,51 \%$ & $2,21 \%$ & $3,18 \%$ \\
\hline Livres de cárie & $30 \%$ & $32,5 \%$ & $27,5 \%$ & $35 \%$ & $13 \%$ \\
\hline
\end{tabular}




\section{Discussão}

O panorama epidemiológico da cárie dentária é caracterizado pelo declínio que vem ocorrendo em diferentes regiões brasileiras. Contribui para esse resultado a fluoretação das águas de abastecimento, a adição do flúor nos dentifrícios no final da década de 1980 ea reforma do SUS, criando condições para ampliação e reorientação das ações em saúde bucal24.

Segundo dados da SABESP, desde 1986 érealizada a fluoretação das águas de abastecimento de Itaí e o heterocontrole do flúor é feito pelo PROÁGUA (Programa de análises laboratoriais nos sistemas públicos de abastecimento de água nos municípios do Estado de São Paulo), apresentando níveis satisfatórios em suas análises com teor de flúor em torno de 0,06ppm. Conforme dados da vigilância sanitária municipal, apenas a zona rural não tem acesso à água tratada.

$M$ artins et al. ${ }^{13}$ observaram, no município de Bilac, São Paulo, queestá ocorrendo declínio dos índices CPOD e aumento da porcentagem de adolescentes livres de cárie, apesar de o município não apresentar fluoretação das águas deabastecimento. Baldani et al. ${ }^{25}$ constataram no Estado do Paraná uma correlação negativa entre 0 CPOD eo percentual dedomicíliosligadosà rede de abastecimento, indicando menores níveis de agravamento nos municípios com maior oferta do serviço de águas. Segundo Nadanovsky ${ }^{26}$, a água fluoretada parece oferecer um benefício adicional, mesmo quando os dentifrícios contêm flúor.

Sales-Peres $\&$ Bastos $^{4}$ em seu estudo na re gião centro-oeste do Estado de São Paulo verificaram que não houve diferença significativa entreo CPOD demunicípios demesmo porte, com ou sem flúor na água, demonstrando o fenômeno da convergência nesta região. Este pode ser justificado pelo efeito halo, queéa exposição dos indivíduos aos diferentes veículos contendo flúor.

Em 1998, o índiceCPOD aos 12 anos de idade no município de Blumenau, Santa Catarina, foi de 1,46, comparativamente o valor foi mais elevado em Porto Feliz, São Paulo de2,20, de2,71 em Rio Claro, São Paulo e de 3,30 em Leme, São Paulo27-30. Gomes et al. ${ }^{1}$ encontraram, no município de Paulínia, São Paulo, CPOD 1,00 no ano 2000. Em Bilac, São Paulo, no ano de 2004 o CPOD encontrado foi de 2,62 14. N este estudo, foi encontrado CPOD de 2,45 nos adolescentes deltaí e, segundo a OM S, o nível decárieéconsiderado baix ${ }^{7}$. M as o valor é elevado quando comparado aos níveis de Paulínia, Blumenau e
Porto Feliz, sendo que estes dados são de anos anteriores.

O declínio dos índices de cárie em vários países foi acompanhado por um processo de polarização da doença numa pequena parcela da população. Permanecendo excluída dos benefícios, seja porque as medidas de saúde coletiva mais importante (água e dentifrício fluorados) não alcançaram ainda esse segmento, seja porque as condições de exclusão social erisco à cárietêm se mantido em níveis extremamente el evados ${ }^{31}$.

Sales-Peres et al. ${ }^{32}$, no município de Bauru, avaliaram o SiC e encontraram o valor de 4,89, considerado dealta prevalência de cárie dentária, como os achados neste estudo, que encontrou 0 valor de 5,08.

Observou-se que $34 \%$ dos adolescentes aos 12 anos de idade de Itaí concentraram $70 \%$ da doença, val ores similares foram encontrados em Pareci N ovo, Rio Grande do Sul ${ }^{16}$.

Isso salienta a importância em se planejar estratégias adequadas para o grupo $\mathrm{SiC}$, visando reduzir a prevalência e a severidade da doença nesse grupo polarizado. Fato este que demonstra o grande valor do emprego deste índice para o planejamento das ações em saúde.

Neste estudo, não foi observada diferença estatisticamente significante no CPOD, quanto ao gênero, bem como em Florianópolis, Santa Catarina ${ }^{33}$.

Os adolescentes residentes na zona rural de I taí apresentaram índice mais elevado (CPOD = $3,18)$ que aqueles que residem na zona urbana $(2,21)$, situação semelhanteà encontrada em Itapetininga e Leme $e^{10,30,34}$. Segundo M ello \& Antunes $^{10}$, isso acontece porque a zona urbana envolve, em geral, padrões médios de renda mais elevados e maior provisão de serviços odontológicos que a zona rural.

A boa cobertura dos serviços odontológicos apresentada pelo componente " $\mathrm{O}$ ", que atingiu $64 \%$ do valor total do CPOD, também foi observada em Blumenau, Leme, Treviso e Itapetininga $^{10,27,30,35 ;}$; diferente deste resultado foi encontrado em São João do Sul, onde o componente mais encontrado foi o " $\mathrm{C}$ " com $51 \%{ }^{35}$.

Diferentes resultados estão descritos na literatura sobre o percentual de livres de cárie encontrados nas regiões brasileiras. No município de Pareci Novo, Rio Grande do Sul, somente $3,22 \%$ dos adolescentes aos 12 anos estavam livres de cárie, um valor considerado baixo, justificado pelos pesquisadores pela ausência de flúor na água de abastecimento público ${ }^{16}$. Por outro lado, em Bauru foi encontrado que $55 \%$ dos ado- 
lescentes não apresentavam experiência de cárie ${ }^{32}$ e no presente estudo o percentual foi de $30 \%$.

Algumas reflexões podem ser elaboradas diante dos resultados obtidos, como a importância da fluoretação das águas de abastecimento aliada ao uso de dentifrícios fluoretados e a implementação de programas educativos e preventivos para pré-escolares. Este tripé pode justificar a baixa prevalência de cárie dentária no grupo estudado.

A ausência de dados sobre as condições de saúde bucal da população pode levar a um planejamento inadequado por parte dos gestores de saúde. Este levantamento epidemiológico foi a primeira pesquisa a ser realizada com adolescentes em Itaí, trazendo à luz do conhecimento problemas eindicadores a serem analisados para incorporação de novas estratégias de atenção à saúde bucal.

Esses resultados poderão nortear a implementação de ações em saúde bucal no município de
Itaí, contribuindo para o planejamento ea al ocação de recursos, em políticas públicas de saúde.

Novas pesquisas devem ser realizadas para confirmar esses achados, quanto à distribuição da doença nos gêneros e localização geográfica.

\section{Conclusões}

Concluiu-se que está ocorrendo a polarização da cárie dentária em adolescentes, aos 12 anos, no município de Itaí. A localização geográfica interferiu nas condições de saúde bucal da população estudada, sendo que não houve diferenças entre os gêneros.

Os problemas bucais se intensificam em uma pequena parcela da população, a qual requer maior atenção odontológica. Os agravos e a desigual dade na distribuição da cárie dentária entre os adolescentes podem ser minimizados por meio da integralidade, contemplando prevenção, promoção e tratamento de saúde bucal.

\section{Colaboradores}

FS Carvalho realizou a coleta de dados, organizou o banco de dados, participou da análise dos resultados e redigiu o artigo. CAP Carvalho participou da coleta dos dados (anotador) e da redação do artigo. JRM Bastos auxiliou no planejamento e condução da pesquisa. JRP Lauris participou da análise estatística. SH C Sales-Peres trabalhou na concepção teórica, na metodologia, na orientação da discussão e na redação do artigo. 


\section{Referências}

1. Gomes PR, Costa SC, Cypriano S, Sousa M LR. Paulínia, São Paulo, Brasil: situação da cárie dentária com relação às metas OM S 2000 e 2010. Cad Saúde Pública 2004; 20(3):866-870.

2. Marshall TA. Dental caries and beverage consumption in young children. Pediatrics 2003; 112(3):184191.

3. Bezerra ACB, Toledo AO. Nutrição, dieta e cárie dentária. In: Kriger L, organizador. Promoção de saúde bucal. São Paulo: Artes M édicas; 2002. p. 43-68.

4. Sales-Peres SHC, Bastos JRM. Perfil epidemiológico de cárie dentária em crianças de 12 anos de idade, residentes em cidades fluoretadas e não fluoretadas, na região Centro-O este do Estado de São Paulo, Brasil. Cad Saúde Pública 2002; 18(5):1281-1288.

5. Narvai PC, Frazão $P$, Castellanos RA. Declínio na experiência de cárie em dentes permanentes de escolares brasileiros no final do século XX. Rev Odontol e Sociedade 1999; 1(1/2):25-29.

6. Brasil. M inistério da Saúde. Levantamento epidemiológico em saúde bucal: Brasil, zona urbana 1986. Brasília: M inistério da Saúde; 1988.

7. World Health Organization. Dental caries levels at 12 years. Geneva: WHO; 1994.

8. Brasil. Ministério da Saúde. Secretaria de Assistência à Saúde. Departamento de Assistência e Promoção à Saúde. Coordenação de Saúde Bucal: $11^{a}$ etapa cárie dental- projeto. Brasília: M inistério da Saúde; 1996.

9. Pinto VG. Saúde bucal: odontologia social e preventiva. São Paulo: Santos; 2000.

10. M ello TRC, Antunes JLF. Prevalência de cárie dentária em escolares da região rural de Itapetininga, São Paulo, Brasil. Cad Saúde Pública 2004; 20(3):829-835.

11. Roncalli AG. Epidemiologia e saúde bucal coletiva: um caminhar compartilhado. Cien Saude Colet 2006; 11(1):105-114.

12. Frias AC, Antunes JLF, Narvai PC. Precisão e validade dos levantamentos epidemiológicos em saúde bucal: cárie dentária na cidade de São Paulo, 2002. Rev Bras Epidemiol 2004; 7(2):144-153.

13. Lopes ES, Bastos JRM . O dontologia preventiva e social. Bauru: Faculdade de Odontologia de Bauru; 1988.

14. Martins RJ, Garbin CAS, Garbin AJI, M oimaz SAS, Saliba O. Declínio da cárie em um município da região noroeste do Estado de São Paulo, Brasil, no período de 1998 a 2004. Cad Saúde Pública 2006; 22(5):1035-1041.

15. Narvai PC, Castellanos RA, Frazão P. Prevalência de cárie em dentes permanentes de escolares do município de São Paulo, SP, 1970-1996. Rev. Saúde Pública 2000; 34(2):196-200.

16. Cardoso L, Rösing C, Kramer P, Costa CC, Costa FiIho LC. Polarização da cárie em município sem água fluoretada. Cad Saúde Pública 2003; 19(1):237-243.

17. Rose G. Sick individuals and sick populations. Int J Epidemiol 1985; 14(1):32-38.

18. Narvai PC, Frazão P, Roncalli AG, Antunes JLF. Cárie dentária no Brasil: declínio, polarização, iniqüidade e exclusão social. Rev Panam Salud Publica 2006; 19(6):385-393.
19. Weyne SC. A construção do paradigma de promoção de saúde - um desafio para as novas gerações. In: Kriger L, organizador. Promoção de saúde bucal. São Paulo: Artes Médicas; 1997. p. 3-26.

20. Brasil. Ministério do Planejamento, Orçamento e Gestão. Instituto Brasileiro de Geografia e Estatística. Estimativa da população brasileira para 2006; 2006 [acessado 2006 jul 15]. Disponível em: http:// www.datasus.gov.br

21. Gueiros LAM, Silva M DP. Inquérito de cárie dentária e perfil alimentar em escolares de 6 a 12 anos de duas populações da região metropolitana do Recife. Odont Clin Cientif 2003; 2(3):201-209.

22. World Health Organization. Oral health surveys: basic methods. $4^{\text {th }}$ ed. Geneva: World Health Organization; 1997.

23. Brasil. M inistério da Saúde. Projeto SB Brasil: condições da saúde bucal da população brasileira no ano 2000: manual do examinador. [Secretaria Políticas de Saúde, Departamento de Atenção Básica, Área Técnica de Saúde Bucal]. Brasília: M inistério da Saúde; 2001.

24. Frazão $P$. Tecnologias em saúde bucal coletiva. In: Botazzo C, Freitas SFT. Ciências sociais e saúde bucal: questões e perspectivas. Bauru: Edusc-U nesp; 1998.

25. Baldani MH, Narvai PC, Antunes JLF. Cárie dentária e condições sócio-econômicas no Estado do Paraná, Brasil, 1996. Cad Saúde Pública 2002; 18(3):755-763.

26. Nadanovsky P. 0 declínio da cárie. In: Pinto VG, organizador. Saúde bucal coletiva. São Paulo: Santos; 2000. p. 341-351.

27. Traebert JL, Peres MA, Galesso ER, Zabot NE, $M$ arcenes W. Prevalência e severidade de cárie dentária em escolares de seis e doze anos de idade. Rev. Saúde Pública 2001; 35(3):283-288.

28. Gushia LL, Rihs LB, Wada RS, Sousa M LR. Cárie dentária em crianças de escolas públicas e privadas do município de Porto Feliz, São Paulo, Brasil. Arqu Odontol 2003; 39(4):257-264.

29. Hoffmann RHS, Cypriano S, Sousa MLR, Wada RS. Experiência de cárie dentária em crianças de escolas públicas e privadas de um município com água fluoretada. Cad Saúde Pública 2004; 20(2):522-528.

30. Tagliaferro EPS, Rihs LB, Sousa MLR. Prevalência de cárie, fluorose dentária e necessidade de tratamento em escolares, Leme, SP. Arqu O dontol 2002; 38(3):213-221.

31. Antunes JLF, Peres MA, Frazão P. Cárie dentária. In: Antunes JLF, Peres MA, organizadores. Epidemiologia da saúde bucal. Rio de Janeiro: Guanabara Koogan; 2006. p.49-67.

32. Sales Peres SHC, M ercadante DCF, Bastos JRM, Pereira AC. Epidemiologia da cárie dentária: distribuição e severidade em população de baixa prevalência. Braz Oral Res 2006; 20(Suppl):38.

33. Freysleben GR, Peres MAA, M arcenes W. Prevalência de cárie e CPOD médio em escolares de doze e treze anos de idade nos anos de 1971 e 1997, região Sul, Brasil. Rev. Saúde Pública 2000; 34(3):304-308. 
34. Irigoyen ME, Luengas IF, Yashine A, M ejia AM, $M$ aupome $G$. Dental caries experience in M exican schoolchildren from rural and urban communities. Int Dent J 2000; 50:41-45.

35. Traebert J, Suárez CS, O nofri DA, M arcenes W. Prevalência e severidade de cárie dentária e necessidade de tratamento odontológico em pequenos municípios brasileiros. Cad Saúde Pública 2002; 18(3):817-821.

Artigo apresentado em 18/01/2007

Versão final apresentada em 09/01/2008 